\title{
Las guías de cuidados preventivos no están integradas aún a la práctica clínica
}

Provision of preventive care unannounced standarized patients.

Hutchison B, Woodward CA, Norman GR, et al. Can Med Assoc J. 1998;158(2):185-93.

\section{Objetivo}

Evaluar las características de la práctica y entrenamiento médicos en relación con los cuidados preventivos, según las guías de la Canadian Task Force (CTF) on the Periodic Health Examination.

\section{Diseño}

Estudio de corte transversal.

\section{Lugar}

Consultorios de Medicina Familiar abiertos a nuevos pacientes en las proximidades de Hamilton, Ontario, Canadá.

\section{Participantes}

Fueron randomizados 125 médicos de un total de 480 médicos de familia que habían respondido una encuesta sobre cuidados preventivos. Un $32,5 \%$ rechazó participar, otro 15,2\% se negó a aceptar pacientes, quedando 62 médicos participantes.

\section{Intervención}

Se evaluó la utilización de practicas preventivas (PP) mediante 4 escenarios estandarizados que fueron actuados por "pacientes" simulados que pretendían iniciar su atención médica. Se eligieron hombres de 48 y 70 años y mujeres de 28 y 52 años, a los que se entrenó según el escenario elegido usando dramatización, cámara Gesell etc. y se les dio información también sobre las PP realizadas 'previamente' . Debían rechazar tacto rectal o vaginal argumentando hemorroides o menstruación y completar un formulario inmediatamente después de la consulta. Dado que muchos médicos realizan su valoración inicial en dos visitas, se aceptó hasta una visita de seguimiento.

\begin{abstract}
Medición de resultados principales
Proporción de utilización de PP con recomendación " $A$ ", " $B$ ", " $C$ ", " $D$ " $y$ " $E$ ", según la fuerza de tareas preventivas de Canadá (CTF)*, realizadas, ofrecidas o aconsejadas. Se utilizaron puntajes estandarizados basados en la implementación de PP "A o B" menos el uso de $P P$ “D o E" para estimar la evaluación de la utilización de cuidados preventivos.
\end{abstract}

\section{Resultados principales}

Los médicos brindaron $65.6 \%$ de las PP "A", 31\% de las "B", $22.4 \%$ de Las " $C$ ", $21.8 \%$ de las " $D$ " y $4.9 \%$ de las "E". La realización de algunas de las "A" fueron: $28 \%$ palpación mamaria, $42 \%$ antitetánica, $87 \%$ evaluación de tabaquismo. De las "B", la evaluación auditiva y visual, consejo sobre uso del cinturón de seguridad y protección solar fueron poco realizadas aunque el $90 \%$ controló la tensiōn arterial. De las " $D$ ", el $30 \%$ solicitó glucemias o análisis de orina y el 13\% radiografía de tórax. La mejor perfomance de cuidados preventivos basados en la evidencia se asoció a la práctica individual (en lugar de grupal) y al pago por cápita o salario (vs. pago por prestación). Este patrón de utilización no se asoció al sexo, certificación en medicina familiar, o formación universitaria (tradicional vs. por problemas [Mc Master\#]) La edad media de los médicos fue de 40 años. Atendían un promedio de 4.8 pacientes/hora y el tiempo insumido por paciente estandarizado fue de $19.3 \mathrm{~min}$.

\section{Conclusiones}

Las guías de PP no se cumplen integralmente a la práctica clínica.

\section{COMENTARIO}

Numerosos estudios de implementación de PP han demostrado que usualmente la mayoría de los pacientes no reciben las PP requeridas para su cuidado ${ }^{1-3}$. Los resultados de este estudio muestran que sólo se brindó un promedio de $41 \%$ de la PP recomendadas por la CTF y un $17 \%$ de las no recomendadas. Dado que la mitad de los médicos respondieron a la encuesta y que de los randomizados sólo participaron la mitad, es probable que el sesgo de selección de médicos motivados sobrevalore la perfomance de cuidados preventivos respecto a la totalidad de los médicos del área. Los estudios que evalúan la relación entre características de la práctica médica y las PP suelen basarse en el autoreporte médico, encuestas a pacientes o revisión de historias clínicas sujetos a sesgos o errores de registro ${ }^{4}$. La medición de las PP por pacientes estandarizados es quizás el mejor método para valorar su utilización en la práctica real 5 , aunque no está completamente exenta de sobrestimar la perfomance dado el conocimiento de los médicos de saberse evaluados. Además, los pacientes estandarizados fueron descubiertos o sospechados en 2 y $20 \%$ de los encuentros respectivamente, esta situación no se asoció con una mejor perfomance preventiva. Por otra parte la perfomance podría estar parcialmente *Ver Glosario \# Currícula universitaria que desarrolla el autoaprendizaje a través de problemas, apoyándose en la medicina basada en la evidencia.

\section{Dr. Agustín Ciapponi}

Unidad de Medicina Familiar y Preventiva. Hospital Italiano de Buenos Aires.

\section{Referencias}

1. Smith Hellen E.; Herbert Carol P.. Preventive practice among primary care physicians in british Columbia: relation to recomendation of the canadian task force on the periodic health examination. Can Med Assoc. J 1993; 149 (12): 1795-1800.

2. Grimshaw Jeremy M., Russell Ian T..Effect of clinical guidelines on medical practice: a sistematic review of rigorous evaluation. Lancet 1993;342:1317-1322.

3. Rubinstein A., Razé A., Kapitowsky K., Maturi G, Botargues M.,et al. Evaluation of Education vs. Education, Audit and feed-back in the Provider Compliance with Preventive Services in the Primary Care Setting. Journal of General Internal Medicine. 1996; Vol 11 Supp 1: 128 (abs).

4. Carney Patricia A., Dietrich Allen J., Freeman Daniel H.,Jr. andNott Leila A.The periodic health examination provided to asintomatic older women:An assessment using standarized patients. Ann.Inter Med. 1993:119:129-135.

5. Lomas Jonathan, Anderson Geoffrey M., Dominick Pierre Karin,et al. Do practice guidelines guide practice? N. Engl. J. Med. 1989;321:1306-11.

6. Mc Phee Stephen J. Adair Bird,Joyce, Jenkins Cristopher N.H., Fordhamm Don. Promoting cancer screening:A randomized controlled trial of three interventions. Arch. Intern. Med. 1989;149:1866-1872.

7. U.S. Public Health Service, Washington D.C. Implementing preventive care. AFP 1994; 50 (No.1): 103-108.

8. Delamonte Tony. Wanted, guidelines that doctors will follow: implementation is the problem. BMJ 1993;307:218. subestimada debido a que la CTF recomienda la realización de PP en forma oportunista en visitas por otros motivos, y en este estudio sólo consideraron a lo sumo dos visitas. Debido a que el diseño de corte ber si la práctica individual y el pago por salario o cápita favorecen la realización de PP. Las diferencias con la práctica grupal y el pago por prestación solo fueron moderadas y con una importante dispersión, por o que deberíamos relativizar estos hallazgos.

dos preventivos aún en lugares como Canadá, con un sistema de salud tio se tornan países donde esto no existe, los hallazgos de este estutales en la estrategia preventiva, es sabido que por si solas no aseguran una mayor adherencia a las PP. Aún cuando se conozcan y acepten (cosa que en realidad tampoco ocurre) $)^{2-6}$ o se utilicen criterios propios adoptados por consenso, ${ }^{2}$ generan pocos cambios de actitud a menos que se acompañen de otras estrategias para superar las barreras de cada práctica en particular. ${ }^{1-7-8}$ 